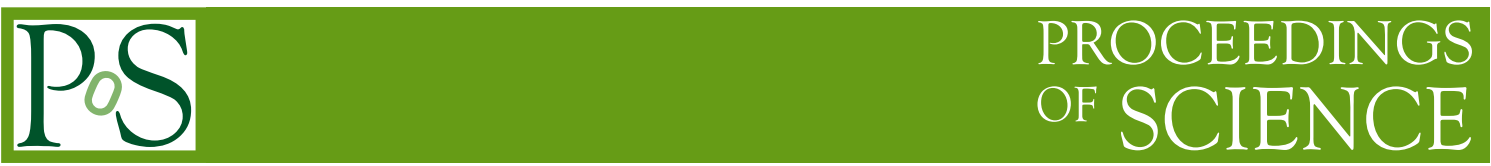

\title{
Cascade Baryon Spectrum from Lattice QCD
}

\author{
Nilmani Mathur ${ }^{\star a}$, John M. Bulava ${ }^{b}$, Robert G. Edwards ${ }^{c}$, Eric Engelson ${ }^{d}$, Bálint \\ Joó $^{c}$, Adam Lichtl ${ }^{e}$ Huey-Wen Lin ${ }^{c}$, Colin Morningstar ${ }^{b}$, David G. Richards ${ }^{c}$, and \\ Stephen J. Wallace ${ }^{d}$
}

(for the Hadron Spectrum Collaboration)

${ }^{a}$ Department of Theoretical Physics, Tata Institute of Fundamental Research, Homi Bhabha

Road, Mumbai 400005, India

${ }^{b}$ Department of Physics, Carnegie Mellon University, Pittsburgh, PA 15213

${ }^{c}$ Thomas Jefferson National Accelerator Facility, Newport News, VA 23606

${ }^{d}$ Department of Physics, University of Maryland, College Park, MD 20742

${ }^{e}$ RIKEN-BNL Research Center, Brookhaven National Laboratory, Upton, NY 11973

E-mail: nilmani@theory.tifr.res.in

\begin{abstract}
A comprehensive study of the cascade baryon spectrum using lattice QCD affords the prospect of predicting the masses of states not yet discovered experimentally, and determining the spin and parity of those states for which the quantum numbers are not yet known. The study of the cascades, containing two strange quarks, is particularly attractive for lattice QCD in that the chiral effects are reduced compared to states composed only of $\mathrm{u} / \mathrm{d}$ quarks, and the states are typically narrow. We report preliminary results for the cascade spectrum obtained by using anisotropic $N_{f}$ $=2$ Wilson lattices with temporal lattice spacing $5.56 \mathrm{GeV}^{-1}$.
\end{abstract}

The XXVI International Symposium on Lattice Field Theory

July 14-192008

Williamsburg, Virginia, USA

\footnotetext{
*Speaker.
} 


\section{Introduction}

A comprehensive understanding of the experimental hadron spectrum from a first principle non-perturbative calculation is one of the main research program for lattice QCD. The lattice hadron spectrum collaboration is mainly focusing on that. Along with nucleon spectrum we are also extracting spectrum for hyperons, and cascade baryons in particular. In this report we present preliminary findings for the cascade baryons by using $N_{f}=2$ anisotropic Wilson action.

Cascade spectrum can be studied comprehensively by lattice QCD and even with quenched lattice QCD. There are a few advantage of studying cascade over studying nucleon, lambda, sigma and delta. First, at low quark mass region, quenched QCD is plagued by the appearance of unphysical ghost states arising out of $\eta^{\prime}$ loop. Since these ghost states are associated with negative correlators, in general, it is very difficult to extract any signal for a physical state. However, since cascades have two strange-mass quarks, presences of such unphysical states are somehow attenuated, although still present and most of the physical states appear as a ground states up to a quite small pion mass region. With dynamical fermions there are no ghost states, but one has to separate out multi-particle decay states from the resonance states. Secondly, due to the presence of two heavier strange quarks, the lattice signal for cascade baryons is much better than that of light quark baryons, such as the nucleon and delta. Thirdly, again due to the presence of two strange quarks, chiral extrapolation for cascade baryons are easier than many other light quark baryons. Beside these, since the decay widths of cascades are small there will be less ambiguity regarding the mixing of states.

\section{Experimental cascade spectrum and lattice opportunity}

In Fig. 1 we have plotted masses (left) and mean Breit-Wigner widths (right) of experimental cascade $(\Xi)$ and omega $(\Omega)$ baryons. In the left figure known $\Xi$ and $\Omega$ states are shown along with their quantum numbers $\left(J^{P}\right)$ and star statuses (s). Except for a few states (ground states in $1 / 2^{+}, 3 / 2^{+}, 3 / 2^{-}$for $\Xi$, and $3 / 2^{+}$for $\Omega$ ) either or both of the quantum numbers of these states are not known. Another interesting aspect of these baryons is that their decay widths are much narrower compare to nucleon states which are shown in right figure. The average width of nucleon states is about $274 \mathrm{MeV}$, whereas for $\Xi$ and $\Omega$ baryons together it is just about $34 \mathrm{MeV}$.

By observing the experimental spectrum, as shown above, it is evident that a comprehensive study of cascades and omegas by lattice QCD will enable us to predict the quantum numbers of many states for which these are still unknown experimentally. Also lattice predicted new states can be helpful for experimental discovery of those states. For example, one can hope to extract a reliable ground state mass for negative parity octet cascade which is still not known. Also, it may be possible to extract a few excited states reliably. For example, it is possible to extract the first excited state in the positive parity octet channel. An indication of such a state will open up the possibility of a Roper like state. That will settle the issue of the quantum numbers of the $\Xi(1690)$, where experimental evidence suggests a state of spin-1/2 [1], but where the parity is still unresolved. Furthermore, a lattice prediction of two states (negative parity ground state and positive parity excited state) will be quite encouraging to search for these states experimentally. The lattice prediction of excited states for spin $3 / 2$ baryons will also settle quantum numbers for a 

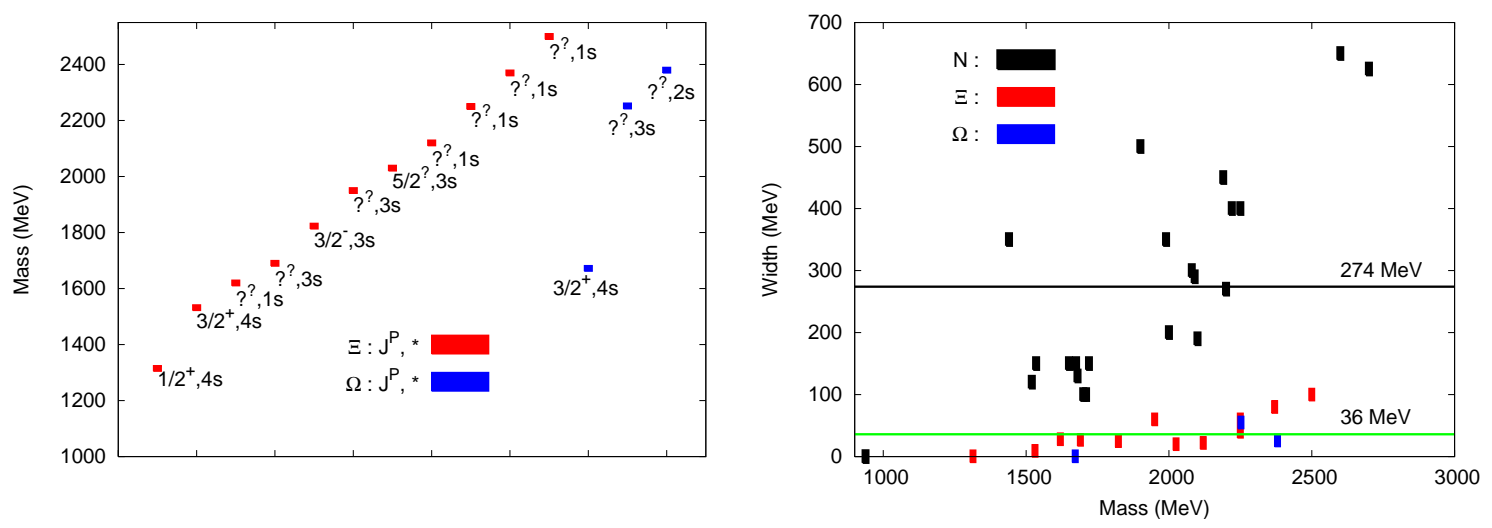

Figure 1: PDG quoted mass (left) and mean Breit-Wigner width (right) of $\Xi$ and $\Omega$ baryons. On the right figure mean Breit-Wigner widths of nucleon resonances are also plotted as a comparison. Combine average widths of $\Xi$ and $\Omega$ 's, and average widths of nucleons are shown by green and black lines respectively.

few states around $1900 \mathrm{MeV}$, and can motivate the experimental search for the properties of such states. Similarly for $\Omega$ baryons, one hopes to predict the negative parity ground state and a positive parity excited state. With our new sophisticated technology comprising group theoretical operators, as discussed below, we hope to predict many other excited states.

\section{Cascade Operators}

As outlined in Ref. [2] for nucleons, we construct cascade operators which transform irreducibly under the symmetries of the lattice i.e., of the octahedral group. In table 1 we show the irreducible representation (irreps) of the lattice octahedral group $\Lambda=\left\{G_{1}, H, G_{2}\right\}$ and the associated continuum angular momentum.

Table 1: The irreducible representation of the octahedral group $\Lambda=\left\{G_{1}, H, G_{2}\right\}$ and the associated continuum angular momentum $J$ up to $\frac{13}{2}$.

\begin{tabular}{|c|c|}
\hline$\Lambda$ & $J$ \\
\hline G1 & $\frac{1}{2} \oplus \frac{7}{2} \oplus \frac{9}{2} \oplus \frac{11}{2} \cdots$ \\
G2 & $\frac{5}{2} \oplus \frac{7}{2} \oplus \frac{11}{2} \oplus \frac{13}{2} \cdots$ \\
H & $\frac{3}{2} \oplus \frac{5}{2} \oplus \frac{7}{2} \oplus \frac{9}{2} \cdots$ \\
\hline
\end{tabular}

In order to build these operators one first constructs the basic building blocks which are covariantly displaced smeared quark fields $\left(\tilde{D}_{j}^{(n)} \tilde{\psi}(x)\right)_{A a \alpha}$ with flavour $A$, colour $a$, and spin $\alpha$. The smeared quark field $\tilde{\psi}$ is displaced $n$ links in the $j$ direction $(j=0, \pm 1, \pm 2, \pm 3)$. It has been observed that both quark smearing and gauge link smearing are necessary to reduce noise and coupling to high energy states. As in our previous studies [3], we use Gaussian quark smearing and stout link smearing. The next step is to construct elemental operators from these displaced single quark operators as

$$
\Phi_{\alpha \beta \gamma ; i j k}^{A B C}(x)=\varepsilon_{a b c}\left(\tilde{D}_{i}^{(n)} \tilde{\psi}(x)\right)_{A a \alpha}\left(\tilde{D}_{j}^{(n)} \tilde{\psi}(x)\right)_{B b \beta}\left(\tilde{D}_{k}^{(n)} \tilde{\psi}(x)\right)_{C c \gamma}
$$


Table 2: The various type of operator, and corresponding quark displacements needed in elemental operators where displacement indices indicate the direction of the gauge-covariant displacement for each quark; $i, j, k=\{0, \pm 1, \pm 2, \pm 3\}$.

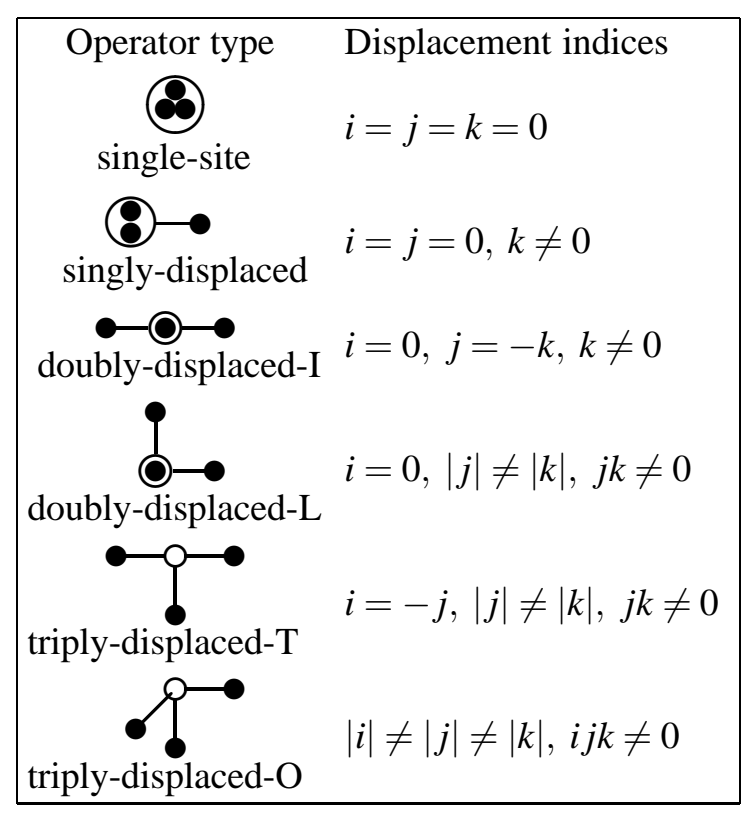

Cascade operators are then constructed by projecting to $I=\frac{1}{2}, I_{3}=\frac{1}{2}$ [3].

We use several different patterns of displacements for our three quark operators, as summarised in Table 2. Taking linear combinations of these elemental operators, we project to the irreps of the octahedral group. The resulting cascade operators for each irreps are shown in table 3 .

Table 3: Type of operators used and their numbers which project into each row of the each irreducible representation of the octahedral group $\Lambda=\left\{G_{1}, H, G_{2}\right\}$ for $\Xi$.

\begin{tabular}{|c|ccc|}
\hline Operator type & G1 & G2 & H \\
\hline Single site & 4 & 0 & 3 \\
Singly displaced & 38 & 14 & 52 \\
Doubly displaced-I & 36 & 12 & 48 \\
Doubly displaced-L & 96 & 96 & 192 \\
Triply displaced & 96 & 96 & 192 \\
\hline Total & 270 & 218 & 487 \\
\hline
\end{tabular}

\section{Pruning of operators}

Not all the operators of table 3 have a substantial overlap with the low-lying spectrum, and it would be very difficult to use all of them in a variational calculation. Indeed, many of them are noisy which can be seen by examining the diagonal elements of the correlation matrix. We have utilised a similar procedure for pruning a best set of operator as discussed in Ref. [3], namely : 
- Check the diagonal effective masses for each irrep and for each parity and sorted those in increasing order of the average jackknife error/signal over the first sixteen non-zero time separations to find out best operators for which signal to noise ratios are larger.

- Using those operators construct normalised correlator matrices in each representation and find their condition numbers.

- For various subset of matrices find a matrix with minimum condition number.

However, in contrast to Ref. [3], we prune each parity channel for each irreps. separately, i.e., positive parity operators are pruned independently of the negative parity operators as their overlap to the physical states could be different.

\section{Results}

We use $24^{3} \times 64$ anisotropic lattices with the anisotropic factor $\xi=3$. We have generated 2 flavour dynamical Wilson gauge configurations, and for fermions we also use Wilson action. We have two set of configurations at temporal lattice spacings $a_{t}^{-1}=5.556 \mathrm{GeV}$ and $5.310 \mathrm{GeV}$. The scale is set with the Sommer parameter, and using this scale the pion masses on these lattices are $416 \mathrm{MeV}$ and $572 \mathrm{MeV}$ respectively. Here we will report results for finer lattice (pion mass at 416 $\mathrm{MeV}$ ) with 860 configurations.

In Fig. 2 we have plotted effective masses for a good pruned operator for each irrep and for both parity channels. The left figure is for the positive parity and the right figure is for the negative parity. As expected, the lowest state is for the positive parity G1 which can be associated with the ground state octet (spin-1/2 $\Xi(1314)$ ). The next lowest state is the positive parity $\mathrm{H}$ which can be of spin $3 / 2$ or spin $5 / 2$. The lowest spin contributing to $G_{2}$ is spin-5/2. We find this mass considerably above the ground state in $\mathrm{H}$; whilst a spin-5/2 state could yield different masses in the two irreps due to finite-lattice-spacing effects, the considerable difference between these ground-states masses leads us to conclude that we are observing a spin-3/2 state in $\mathrm{H}$, and a spin-5/2 state in $G_{2}$.

We are also able to extract good signals for negative parity states which are, in general, difficult to obtain. In Fig. 3 (right), we plot effective masses for the negative parity ground state of G1 for a pruned operator along with the commonly used local negative parity operator. As one can observe that our pruned operator has much better overlap than the other. We believe that due to bigger size of this state one needs an extended operator, and since we have included those, our pruned operator has bigger overlap to this state. The spin identification, as argued above, for negative parity states is not possible as all of them almost overlap to each other (figure 2, right). Another interesting point to note is that for G2, the parity of the ground state is negative as shown in Fig. 3 (left). If one can associate the ground state of G2 with a spin 5/2 state, as argued above, then this result suggests that for spin $5 / 2$ cascade the ground state is for the negative parity, which is in opposite ordering than the parity ordering in spin-1/2 and spin-3/2 baryons.

At a lower pion mass one expects to observe signal for multi-hadron decay states along with resonance states. For example, the $N^{*}(1535)$ decays into a nucleon and pion, and similarly the negative parity spin $1 / 2$ cascade will decay to a multi-hadron state of positive parity cascade and a pion in a S-wave. In Fig. 4 we plotted effective masses of ground state of irreps $G_{1}$ (left) and $H$ (right). The horizontal lines are the sums of the positive parity ground state mass and pion 

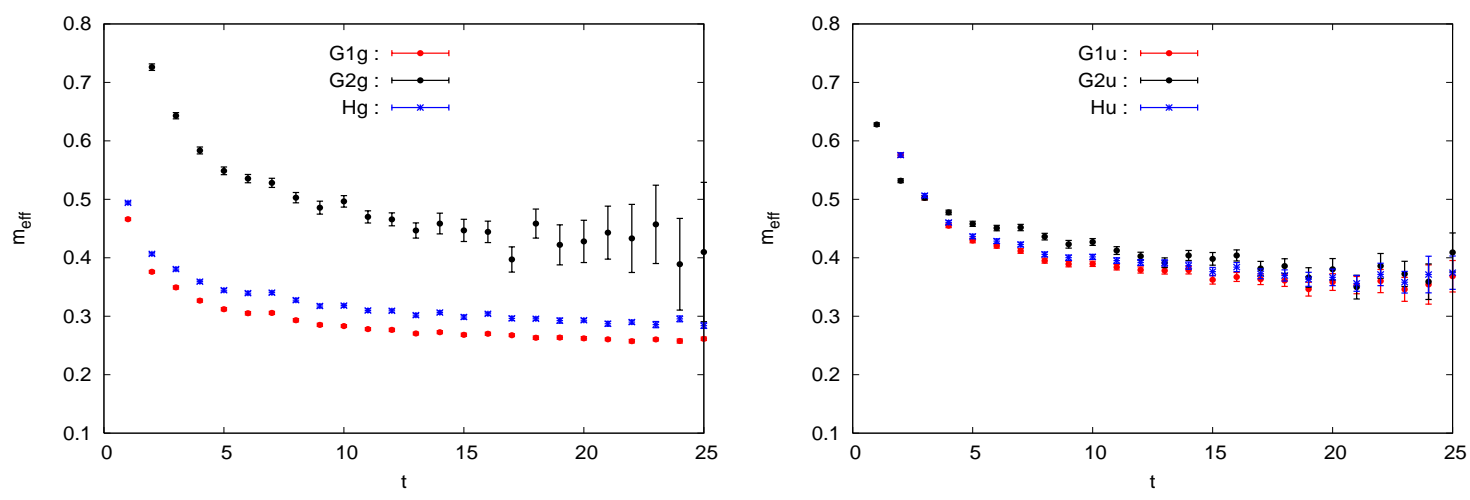

Figure 2: Effective masses of positive (left) and negative (right) parity ground state cascade baryons for various irreps for $24^{3} \times 64 N_{f}=2$ lattice QCD data at $m_{\pi}=400 \mathrm{MeV}$.
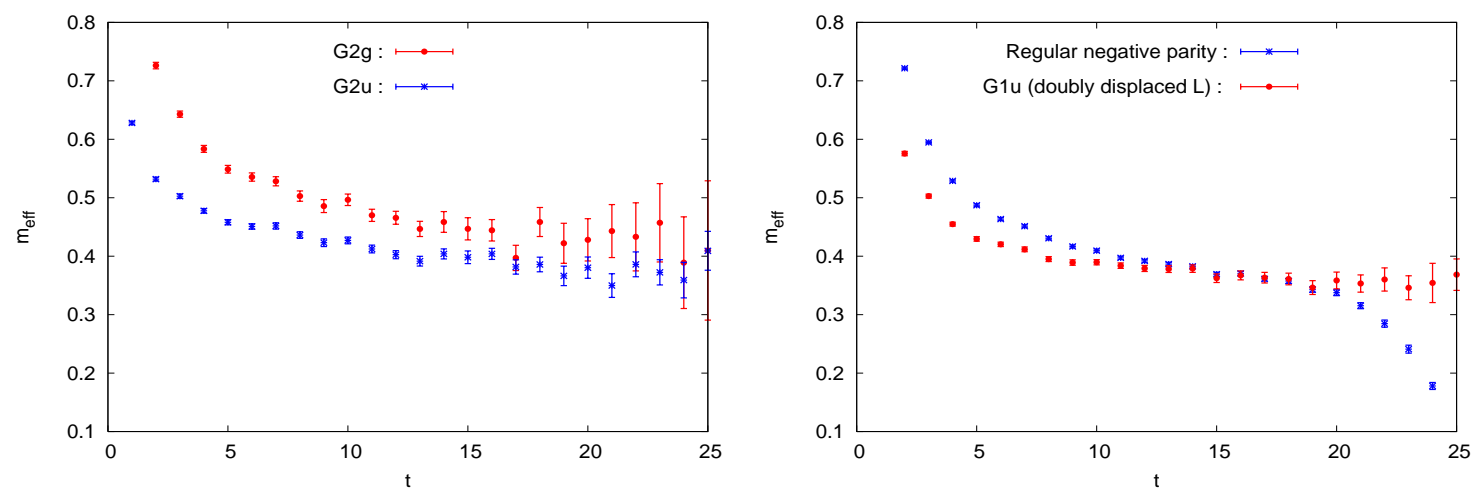

Figure 3: Left : A representative effective mass plot for G2 channel showing that the ground state negative parity mass is lower than that of the ground state positive parity. This parity ordering is opposite to what had been seen in other channels. Right : A comparison of effective masses obtained from an extended operator and most used regular local operator for negative parity G1 channel.

mass, corresponding to the non-interacting cascade-pion state in S-wave. Since these lines are overlapping with the negative parity observed states we argue that the observed states are either multi-hadron states or a mixture of multi-hadron and resonance states.

\section{Conclusions and outlook}

Given the incomplete experimental picture for cascade baryons, and a comprehensive lattice study is demanded, we have initiated such a calculation by using operators which transform as irreps of the octahedral group. Here we have showed our preliminary results for $N_{f}=2$ Wilson fermions at pion mass $416 \mathrm{MeV}$. A preliminary analysis show that we are able to extract clear signals for ground states for each irrep and for each parity. Unlike the case when using only local operators, the use of extended operators enables us to extract signals for negative-parity states unambiguously. We also believe that, at $416 \mathrm{MeV}$ pion, the negative parity ground state extracted for irreps $\mathrm{G} 1$ and $\mathrm{H}$ are probably those of $\Xi-\pi$ multi-hadron scattering states into which the resonance states can decay. For the irrep G2, the negative parity state is lower than that of the positive parity, which is not common for low lying baryons. This preliminary study also suggests 

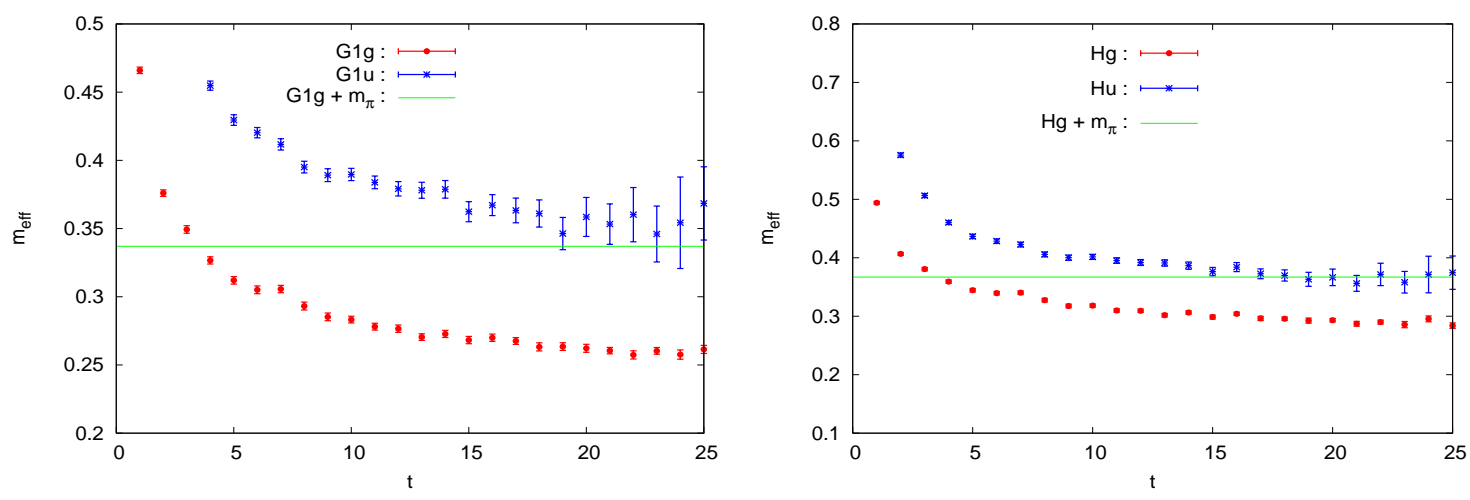

Figure 4: Effective masses of ground state $G_{1}$ (left) and $H$ (right) irreps. The horizontal lines are sum of the positive parity ground state asses and pion mass which indicate that in these channels the observed negative parity states are probably be the $\mathrm{S}$-wave scattering states.

that the observed positive parity ground state for irrep $\mathrm{H}$ has spin 3/2, whilst that for $\mathrm{G} 2$ has spin $5 / 2$. However, this needs to be confirmed for other quark masses and then taking continuum limit. Our current studies are aimed at carefully delineating the single- and multi-particle states before we attempt to proceed to extract the resonance behaviour at lower pion mass We will soon carry out full variational study to extract excited states as was performed for ref. [4] at this pion mass and at a higher pion mass. Our collaboration has begun to generate a series of gauge configurations for $2+1$ dynamical quark flavours for various lattice spacings and volumes [5]. We will be utilising these lattices for extracting cascade spectrum along with other hadrons.

\section{Acknowledgements}

This work was done using the Chroma software suite [6] on clusters at Jefferson Laboratory using time awarded under the USQCD Initiative. This research used resources of the National Center for Computational Sciences at Oak Ridge National Laboratory, which is supported by the Office of Science of the U.S. Department of Energy under the US DOE INCITE 2007 Program. This research was supported in part by the National Science Foundation through Teragrid Resources provided by the San Diego Supercomputing Center (Blue Gene). The research of N.M. is supported under grant No. DST-SR/S2/RJN-19/2007, India.

\section{References}

[1] V. Ziegler, Proc. of the 11th Inter Conf. on Meson-Nucleon. Physics (MENU 2007), pp 309.

[2] S. Basak, et. al, Phys. Rev. D.72, 074501 [arXiv:hep-lat/0508018]; S. Basak, et. al, Phys. Rev. D.72, 094506 [arXiv:hep-lat/0506029].

[3] A. Lichtl, hep-lat/0609019.

[4] J. Bulava et al, arXiv:0810.0253.

[5] H.-W. Lin et al, arXiv:0810.3588.

[6] R. G. Edwards, B. Joó, Nucl. Phys. Proc. Suppl. 140, 832 [arXiv:hep-lat/0409003]. 